\title{
Introducing the theory of bonds for Stewart Gough platforms with self-motions ${ }^{0}$
}

\author{
Georg Nawratil \\ Institute of Discrete Mathematics and Geometry \\ Vienna University of Technology \\ Vienna 1040, Austria \\ Email: nawratil@geometrie.tuwien.ac.at
}

We transfer the basic idea of bonds, introduced by Hegedüs, Schicho and Schröcker for overconstrained closed chains with rotational joints, to the theory of self-motions of parallel manipulators of Stewart Gough type. Moreover we present some basic facts and results on bonds and demonstrate the potential of this theory on the basis of several examples. As a by-product we give a geometric characterization of all $S G$ platforms with a pure translational self-motion and of all spherical 3-dof RPR manipulators with self-motions.

\section{Introduction}

The theory of bonds was introduced by Hegedüs, Schicho and Schröcker in [1] ${ }^{1}$ as a new means for the analysis of overconstrained closed linkages with revolute joints ( $R$ joints). In the following we give only a very tight summary of this theory in order to sketch its basic idea:

A dual unit-quaternion $\underline{\mathbf{h}}:=\left(e_{0}, e_{1}, e_{2}, e_{3}, f_{0}, f_{1}, f_{2}, f_{3}\right)$ describes a rotation in the Euclidean 3 -space, if $f_{0}=0$ holds and $e_{1}=e_{2}=e_{3}=0$ does not hold. ${ }^{2}$ Then $\underline{\mathbf{t}}-\underline{\mathbf{h}}$ yields a 1-parametric rotation around a fixed axis a with $\underline{\mathbf{t}}=(t: 0: 0$ : $0: 0: 0: 0: 0)$ and $t \in \mathbb{R}$.

Now we consider a composition of consecutive rotations, which can be written as:

$$
\left(\underline{\mathbf{t}}_{1}-\underline{\mathbf{h}}_{1}\right) \circ\left(\underline{\mathbf{t}}_{2}-\underline{\mathbf{h}}_{2}\right) \circ \ldots \circ\left(\underline{\mathbf{t}}_{n}-\underline{\mathbf{h}}_{n}\right)
$$

where $\circ$ denotes the product of dual quaternions. If there exists a set $K:=\left\{\left(t_{1}, \ldots, t_{n}\right)\right\}$ of dimension one in a way that Eq. (1) yields an element of $\mathbb{R} \backslash\{0\}$ then the $n$ rotational axes $\mathrm{a}_{1}, \ldots, \mathrm{a}_{n}$ associated with $\underline{\mathbf{h}}_{1}, \ldots, \underline{\mathbf{h}}_{n}$ form a closed $n \mathrm{R}$ linkage with one degree of freedom.

Now the set of bonds is defined as those elements of the so-called configuration curve $K$, which imply that Eq. (1)

\footnotetext{
${ }^{0}$ Dedicated to my newborn daughter and her mother on the occasion of her birth.

${ }^{1}$ Recently an extended version of this paper was published (cf. [2]).

${ }^{2}$ For $e_{1}=e_{2}=e_{3}=0$ the rotation degenerates into a translation. This case is excluded.
}

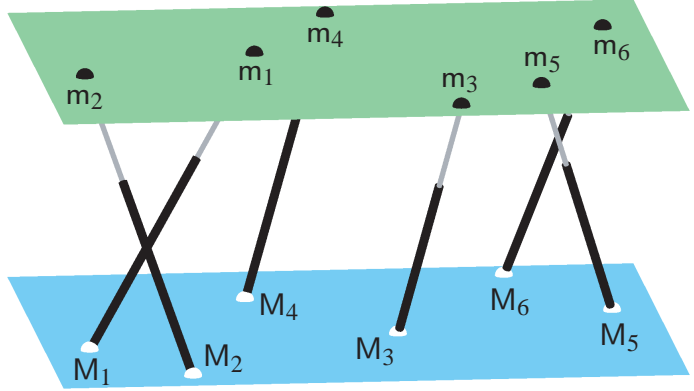

Fig. 1. A SG manipulator with planar platform and planar base $\left(C_{i}=c_{i}=0\right.$ for $\left.i=1, \ldots, 6\right)$ is called planar SG manipulator.

equals the exceptional number 0 . Therefore bonds are the points on the complex configuration curve at some degenerate infinity, which contain a lot of information regarding the geometry of the overconstrained closed chain. For more details we refer to [1].

Remark 1. For a better understanding of the concept of bonds we offer the following well known analogy: To a curve $k$ in Euclidean 3-space, we associate the points of $k$ at infinity. These ideal points correspond with the bonds. If this set is a singleton, then the curve is a line in direction of the ideal point. If it consists of two points located on the absolute conic, then the curve is circular, and so on.

\subsection{Stewart Gough manipulator}

The geometry of a Stewart Gough (SG) platform is given by the six base anchor points $M_{i}$ with coordinates $\mathbf{M}_{i}:=$ $\left(A_{i}, B_{i}, C_{i}\right)^{T}$ with respect to the fixed system and by the six platform anchor points $\mathrm{m}_{i}$ with coordinates $\mathbf{m}_{i}:=\left(a_{i}, b_{i}, c_{i}\right)^{T}$ with respect to the moving system (for $i=1, \ldots, 6$ ). Each pair $\left(\mathrm{M}_{i}, \mathrm{~m}_{i}\right)$ of corresponding anchor points is connected by a SPS-leg, where only the prismatic joint $(\mathrm{P})$ is active and the spherical joints (S) are passive (cf. Fig. 1).

If the geometry of the manipulator is given as well as the six leg lengths, the SG platform is generically rigid. But 
under particular conditions the manipulator can perform a $d$-parametric motion $(d>0)$, which is called self-motion. Note that such motions are also solutions to the still unsolved problem posed by the French Academy of Science for the Prix Vaillant of the year 1904, which is also known as Borel Bricard problem (cf. [3-5]) and reads as follows:

"Determine and study all displacements of a rigid body in which distinct points of the body move on spherical paths."

It is already known that manipulators, which are singular in every possible configuration, possess self-motions in each pose (over $\mathbb{C}$ ). These so-called architecturally singular SG platforms are well studied and classified (for the planar case we refer to [6-9] and for the non-planar case see $[10,11])$. Until now only few self-motions of non-architecturally singular SG platforms are known as their computation is a very complicated task. To the best knowledge of the author, a complete and detailed review of these self-motions was given in [12].

In Section 2 we transfer the basic idea of bonds to parallel manipulators of SG type and explain how these bonds can be computed. In Section 3 we present some basic facts, results and examples on bonds of SG platforms with selfmotions. Moreover we characterize all SG platforms with pure translational self-motions, as these self-motions are not covered by the theory of bonds. In the Sections 4 and 5 we demonstrate the potential of bond theory on the basis of two interesting tasks. Finally we close the paper with conclusions and an outlook to future research.

\section{Bonds of parallel manipulators of SG type}

We start with the direct kinematic problem of parallel manipulators of SG type. Due to the result of Husty [13], it is advantageous to work with Study parameters, which are nothing else than homogenized dual unit-quaternions; i.e. $\left(e_{0}: e_{1}: e_{2}: e_{3}: f_{0}: f_{1}: f_{2}: f_{3}\right)$. Note that the first four homogeneous coordinates $\left(e_{0}: e_{1}: e_{2}: e_{3}\right)$ are the so-called Euler parameters.

Now all real points of the 7-dimensional Study parameter space $P^{7}$, which are located on the so-called Study quadric $\Psi: \sum_{i=0}^{3} e_{i} f_{i}=0$, correspond to an Euclidean displacement with exception of the 3-dimensional subspace $E$ of $\Psi$ given by $e_{0}=e_{1}=e_{2}=e_{3}=0$, as its points cannot fulfill the condition $N \neq 0$ with $N=e_{0}^{2}+e_{1}^{2}+e_{2}^{2}+e_{3}^{2}$. The translation vector $\mathbf{t}:=\left(t_{1}, t_{2}, t_{3}\right)^{T}$ and the rotation matrix $\mathbf{R}:=\left(r_{i j}\right)$ of the corresponding Euclidean displacement $\mathbf{R x}+\mathbf{t}$ are given by:

$$
\begin{aligned}
& t_{1}=2\left(e_{0} f_{1}-e_{1} f_{0}+e_{2} f_{3}-e_{3} f_{2}\right), \\
& t_{2}=2\left(e_{0} f_{2}-e_{2} f_{0}+e_{3} f_{1}-e_{1} f_{3}\right), \\
& t_{3}=2\left(e_{0} f_{3}-e_{3} f_{0}+e_{1} f_{2}-e_{2} f_{1}\right),
\end{aligned}
$$

and

$\mathbf{R}=\left(\begin{array}{ccc}e_{0}^{2}+e_{1}^{2}-e_{2}^{2}-e_{3}^{2} & 2\left(e_{1} e_{2}-e_{0} e_{3}\right) & 2\left(e_{1} e_{3}+e_{0} e_{2}\right) \\ 2\left(e_{1} e_{2}+e_{0} e_{3}\right) & e_{0}^{2}-e_{1}^{2}+e_{2}^{2}-e_{3}^{2} & 2\left(e_{2} e_{3}-e_{0} e_{1}\right) \\ 2\left(e_{1} e_{3}-e_{0} e_{2}\right) & 2\left(e_{2} e_{3}+e_{0} e_{1}\right) & e_{0}^{2}-e_{1}^{2}-e_{2}^{2}+e_{3}^{2}\end{array}\right)$ if the normalizing condition $N=1$ is fulfilled. All points of the complex extension of $P^{7}$, which cannot fulfill this normalizing condition, are located on the so-called exceptional quadric $N=0$. It can easily be seen by differentiation that a point of the exceptional quadric is singular, if and only if it belongs to $E$. Therefore $N=0$ is a cone with vertex $E$.

By using the Study parametrization of Euclidean displacements the condition that the point $\mathrm{m}_{i}$ is located on a sphere centered in $\mathrm{M}_{i}$ with radius $R_{i}$ is a quadratic homogeneous equation according to Husty [13]. This so-called sphere condition $\Lambda_{i}$ has the following form:

$$
\begin{aligned}
\Lambda_{i}: & \left(a_{i}^{2}+b_{i}^{2}+c_{i}^{2}+A_{i}^{2}+B_{i}^{2}+C_{i}^{2}-R_{i}^{2}\right) N \\
& -2\left(a_{i} A_{i}+b_{i} B_{i}+c_{i} C_{i}\right) e_{0}^{2}-2\left(a_{i} A_{i}-b_{i} B_{i}-c_{i} C_{i}\right) e_{1}^{2} \\
& +2\left(a_{i} A_{i}-b_{i} B_{i}+c_{i} C_{i}\right) e_{2}^{2}+2\left(a_{i} A_{i}+b_{i} B_{i}-c_{i} C_{i}\right) e_{3}^{2} \\
& +4\left(c_{i} B_{i}-b_{i} C_{i}\right) e_{0} e_{1}-4\left(c_{i} A_{i}-a_{i} C_{i}\right) e_{0} e_{2} \\
& +4\left(b_{i} A_{i}-a_{i} B_{i}\right) e_{0} e_{3}-4\left(b_{i} A_{i}+a_{i} B_{i}\right) e_{1} e_{2} \\
& -4\left(c_{i} A_{i}+a_{i} C_{i}\right) e_{1} e_{3}-4\left(c_{i} B_{i}+b_{i} C_{i}\right) e_{2} e_{3} \\
& +4\left(a_{i}-A_{i}\right)\left(e_{0} f_{1}-e_{1} f_{0}\right)+4\left(b_{i}-B_{i}\right)\left(e_{0} f_{2}-e_{2} f_{0}\right) \\
& +4\left(c_{i}-C_{i}\right)\left(e_{0} f_{3}-e_{3} f_{0}\right)+4\left(a_{i}+A_{i}\right)\left(e_{3} f_{2}-e_{2} f_{3}\right) \\
& +4\left(b_{i}+B_{i}\right)\left(e_{1} f_{3}-e_{3} f_{1}\right)+4\left(c_{i}+C_{i}\right)\left(e_{2} f_{1}-e_{1} f_{2}\right) \\
& +4\left(f_{0}^{2}+f_{1}^{2}+f_{2}^{2}+f_{3}^{2}\right)=0
\end{aligned}
$$

Now the solution of the direct kinematics over $\mathbb{C}$ can be written as the algebraic variety $V$ of the ideal $\mathscr{I}$ spanned by $\Psi, \Lambda_{1}, \ldots, \Lambda_{6}, N=1$. In general $V$ consists of a discrete set of points with a maximum of 40 elements.

After the removal of the $n$-th leg we end up with a 5-legged manipulator, which has at least a 1-parametric self-motion (over $\mathbb{C}$ ). Its solution for the direct kinematics is given by the algebraic variety $V_{n}$ of the ideal $\mathscr{I}_{n}$ spanned by $\Psi, \Lambda_{i}, \Lambda_{j}, \Lambda_{k}, \Lambda_{l}, \Lambda_{m}, N=1$ with pairwise distinct $i, j, k, l, m, n \in\{1, \ldots, 6\}$. Note that $\mathscr{I}_{n}$ is a subideal of $\mathscr{I}$.

In the following we give a rough idea of bonds of 5legged manipulators: We consider the algebraic motion of the mechanism, which are the points on the Study quadric that the constraints define; i.e. the common points of the six quadrics $\Psi, \Lambda_{i}, \Lambda_{j}, \Lambda_{k}, \Lambda_{l}, \Lambda_{m}$. Now the points of the algebraic motion with $N \neq 0$ equal the kinematic image of the algebraic variety $V_{n}$. But we can also consider the points of the algebraic motion, which belong to the exceptional cone $N=0$. An exact definition of these so-called bonds is given within the next section.

\subsection{Direct kinematics of 5-legged manipulators}

Based on the algorithm of Husty [13] for solving the direct kinematics of SG manipulators we sketch the general procedure for computing the forward kinematics of 5-legged parallel manipulators of SG type. In the following we also use the equation $\Delta_{j, i}$, which denotes the difference $\Lambda_{j}-\Lambda_{i}$ of two sphere constraints. Note that $\Delta_{j, i}$ is only linear in $f_{0}, \ldots, f_{3}$. 
The five linear equations $\Psi, \Delta_{j, i}, \Delta_{k, i}, \Delta_{l, i}, \Delta_{m, i}$ in $f_{0}, \ldots, f_{3}$ only have a solution, if the determinant of the augmented coefficient matrix $(5 \times 5$ matrix $)$ of this linear system with respect of $f_{0}, \ldots, f_{3}$ equals zero. From this determinant we can factor out $N$ and we remain with a polynomial $F_{n}$ of degree four in the Euler parameters. This $F_{n}$ can also be seen as a quartic surface $\Phi_{n}$ in the Euler parameter space $P^{3}$, which is a projective 3 -space.

Remark 2. Due to e.g. $\Delta_{j, k}=\Delta_{j, i}-\Delta_{k, i}$ and $\Delta_{k, i}=-\Delta_{i, k}$, the ideal $\mathscr{J}:=\left\{\Psi, \Delta_{j, i}, \Delta_{k, i}, \Delta_{l, i}, \Delta_{m, i}\right\}$ is also spanned by $\Psi, \Delta_{i, k}, \Delta_{j, k}, \Delta_{l, k}, \Delta_{m, k}$. Now $F_{n}$ is the algebraic variety of the elimination-ideal of $\mathscr{J}$ with respect to $f_{0}, \ldots, f_{3}$. As the elimination of $f_{0}, \ldots, f_{3}$ is done linearly, $F_{n}$ does not depend on the choice of $i \in\{1, \ldots, 6\} \backslash\{n\}$.

Moreover we can also solve the subset $\mathscr{S}_{m}$ of equations $\Psi, \Delta_{j, i}, \Delta_{k, i}, \Delta_{l, i}$ with pairwise distinct $i, j, k, l, m \in$ $\{1, \ldots, 6\} \backslash\{n\}$ for $f_{0}, \ldots, f_{3}$ and plug the obtained solutions into $\Lambda_{i}$. The numerator of the resulting expression is a polynomial $G_{m}$ of degree eight in the Euler parameters, which can also be seen as an octic surface $\Gamma_{m}$ in the Euler parameter space $P^{3}$.

Remark 3. $G_{m}$ is the algebraic variety of the eliminationideal of $\Psi, \Lambda_{i}, \Lambda_{j}, \Lambda_{k}, \Lambda_{l}$ with respect to $f_{0}, \ldots, f_{3}$. As the elimination of $f_{0}, \ldots, f_{3}$ is again done linearly, $G_{m}$ does not depend on the choice of $i \in\{1, \ldots, 6\} \backslash\{n, m\}$.

All points of $\Gamma_{m}$ with $N \neq 0$ and $Q_{m} \neq 0$ can be extended to solutions of the direct kinematics of the 4-legged manipulator, which is obtained from the 5-legged one by removing the $m$-th leg. Note that $Q_{m}=0$ is a quadric in the Euler parameter space $P^{3}$, which equals the set of orientations, where the denominator of the $f_{i}$ 's vanishes.

We denote the set of common points of the quartic $\Phi_{n}$ and the five octics $\Gamma_{i}, \Gamma_{j}, \Gamma_{k}, \Gamma_{l}, \Gamma_{m}$ by $K_{n}$. If a point $\mathrm{Q}$ of $K_{n}$ is located on all five quadrics $Q_{i}=Q_{j}=Q_{k}=Q_{l}=Q_{m}=0$, then the back-substitution in all solutions for $f_{0}, \ldots, f_{3}$ yields a contradiction (division by zero). But this already implies for the orientation determined by $\mathrm{Q}$ that the rank of the coefficient matrix of the linear system $\Psi, \Delta_{j, i}, \Delta_{k, i}, \Delta_{l, i}, \Delta_{m, i}$ is less than 4 . Therefore this system lets at least one of the Study parameters $f_{0}, \ldots, f_{3}$ undetermined. As a consequence the only remaining equation (any sphere condition of the involved five legs) can be solved for this Study parameter. Hence the point $\mathrm{Q}$ corresponds with multiple solutions of the direct kinematics (which have the same orientation). Therefore each point of $K_{n}$ can be extended to at least one solution of the forward kinematics problem (over $\mathbb{C}$ ). Summed up we can state that the points of $K_{n}$ with $N \neq 0$ are the projection of $V_{n}$ into the Euler parameter space $P^{3}$, which is induced by the elimination of $f_{0}, \ldots, f_{3}$.

Clearly the kernel of this projection equals the group of translational motions. As a consequence a component of $V_{n}$, which corresponds to a pure translational motion over $\mathbb{C}$ (i.e. $e_{i}=\lambda g_{i}$ where $g_{i}$ are constants $\in \mathbb{C}$ for $i=0, \ldots, 3$ with $g_{0}^{2}+g_{1}^{2}+g_{2}^{2}+g_{3}^{2} \neq 0$ and $\left.\lambda \in \mathbb{C} \backslash\{0\}\right)$, is projected to a single point $\mathrm{O}$ of the Euler parameter space $P^{3}$. As the intersection of $O$ and $N=0$ equals $\varnothing$, we have to restrict ourselves to non-translational motions ${ }^{3}$ for the following definition of bonds, which is similar to Definition 2 of [1]:

Definition 1. For a 5-legged parallel manipulator of $S G$ type the set $\mathscr{B}_{n}$ of bonds is defined as:

$$
\begin{aligned}
\mathscr{B}_{n}:= & \operatorname{ZarClo}\left(V_{n}^{\star}\right) \cap\left\{\left(e_{0}: \ldots: f_{3}\right) \in P^{7} \mid\right. \\
& \left.\Psi, \Lambda_{i}, \Lambda_{j}, \Lambda_{k}, \Lambda_{l}, \Lambda_{m}, N=0\right\},
\end{aligned}
$$

with pairwise distinct $i, j, k, l, m, n \in\{1, \ldots, 6\} . \quad V_{n}^{\star}$ denotes the variety $V_{n}$ after the removal of all components, which correspond to pure translational motions. Moreover $\operatorname{ZarClo}\left(V_{n}^{\star}\right)$ is the Zariski closure of $V_{n}^{\star}$; i.e. the zero locus of all algebraic equations that also vanish on $V_{n}^{\star}$.

Therefore the set of bonds $\mathscr{B}_{n}$ are the exceptional points $(N=0)$ of the algebraic motion, which are limits of nontranslational motions. Due to this definition of $\mathscr{B}_{n}$, the set of bonds can easily be computed by intersecting $K_{n}$ with $N=0$, which can generally be done as follows:

We eliminate an Euler parameter $e_{s}$ by computing the resultant $U_{n}:=\operatorname{Res}\left(F_{n}, N, e_{s}\right)$ of $F_{n}$ and $N$ with respect to $e_{s}$. Beside this polynomial, we can also calculate the resultants $L_{x}:=\operatorname{Res}\left(G_{x}, N, e_{s}\right)$ with $x \in\{i, j, k, l, m\}$. We eliminate a further Euler parameter $e_{t}$ by computing $W_{x}:=$ $\operatorname{Res}\left(U_{n}, L_{x}, e_{t}\right)$. Then the greatest common divisor $G C D_{n}$ of these five equations $W_{i}, W_{j}, W_{k}, W_{l}, W_{m}$ corresponds with the intersection points of $K_{n}$ and $N=0$. Therefore each solution of $G C D_{n}$ implies a bond, which can be computed stepwise by back-substitution (cf. Example 1).

\subsection{Bonds of SG platforms}

Based on Definition 1, we can give the definition of bonds for a SG platform as follows:

Definition 2. For a $S G$ manipulator the set $\mathscr{B}$ of bonds is defined as $\mathscr{B}:=\bigcap_{n=1}^{6} \mathscr{B}_{n}$, where $\mathscr{B}_{n}$ denotes the set of bonds of the 5-legged manipulator after removal of the $n$-th leg.

Due to Definition 2, the set $\mathscr{B}$ equals the common points of $K_{1}, \ldots, K_{6}$ and $N=0$. Therefore these points correspond to common factors of $G C D_{1}, \ldots, G C D_{6}$. Note that it is sufficient to compute the common factors of $G C D_{i}$ and $G C D_{j}$ for $i \neq j$ as all six legs of the manipulator are already involved. But the computation of bonds can further be simplified due to the following consideration:

The common points of $K_{1}, \ldots, K_{6}$ are contained within the six quartic surfaces $\Phi_{1}, \ldots, \Phi_{6}$. Their corresponding equations $F_{1}, \ldots, F_{6}$ can be generated within the ideal

$$
\mathscr{I}_{0}:=\left\{\Psi, \Delta_{j, i}, \Delta_{k, i}, \Delta_{l, i}, \Delta_{m, i}, \Delta_{n, i}\right\}
$$

with pairwise distinct $i, j, k, l, m, n \in\{1, \ldots, 6\}$.

We denote the set of common points of $\Phi_{1}, \ldots, \Phi_{6}$ by $K_{0}$. Note that the algebraic degree of $K_{0}$ is lower than the

${ }^{3}$ Note that this is an analogy to the theory of bonds of overconstrained closed chains (cf. footnote 2 ). 
degree of $K_{1}, \ldots, K_{6}$, as it is determined by the intersection of only quartic surfaces. In the general case, the common points of $K_{0}$ and $N=0$ can be computed as follows:

We eliminate an Euler parameter $e_{S}$ by computing the resultant $U_{i}:=\operatorname{Res}\left(F_{i}, N, e_{s}\right)$. Then we eliminate a further Euler parameter $e_{t}$ by calculating $W_{i, j}:=\operatorname{Res}\left(U_{i}, U_{j}, e_{t}\right)$ for pairwise distinct $i, j \in\{1, \ldots, 6\}$. Now the greatest common divisor $G C D_{0}$ of these equations $W_{i, j}$ corresponds with the intersection points of $K_{0}$ and $N=0$.

Due to the lower algebraic degree of $G C D_{0}$, we recommend to compute $\mathscr{B}$ by using the common factors of $G C D_{0}$ and $G C D_{i}$ for an arbitrary $i \in\{1, \ldots, 6\}$. This is already sufficient, as again all six legs of the manipulator are involved. Finally it should be noted that in the general case there does not exist a common factor of $G C D_{0}$ and $G C D_{i}$; i.e. $\mathscr{B}=\varnothing$ holds (cf. Example 4).

Remark 4. There are two special cases, where the given algorithm has to be modified as the linear system for the solution of $f_{0}, \ldots, f_{3}$ is always linear dependent. According to page 513 of [14], these cases are as follows:

1. the platform and the base are planar and the anchor points are related within an affinity,

2. the platform and the base are congruent.

An algorithm for these cases is given within Example 5.

The theory of bonds is based on the following fundamental theorem:

Theorem 1. The set $\mathscr{B}$ of bonds depends on the geometry of the $S G$ manipulator and not on the leg lengths $R_{1}, \ldots, R_{6}$.

Proof: We consider $K_{n}$ in the Euler parameter space $P^{3}$. Beside the geometry of the corresponding 5-legged manipulator, $K_{n}$ depends on the leg lengths $R_{i}, R_{j}, R_{k}, R_{l}, R_{m}$ with pairwise distinct $i, j, k, l, m \in\{1, \ldots, 6\} \backslash\{n\}$. But the intersection points of $K_{n}$ and $N=0$ are independent of these leg lengths as theses variables do not appear within the polynomials $L_{i}, L_{j}, L_{k}, L_{l}, L_{m}, U_{n}$. The reason for this is that the coefficients of the leg lengths within the equations $G_{i}, G_{j}, G_{k}, G_{l}, G_{m}, F_{n}$ contain the factor $N$. As a consequence the Euler parameters of the bonds do not depend on $R_{i}, R_{j}, R_{k}, R_{l}, R_{m}$.

After back-substitution of the obtained Euler parameters into $\Lambda_{i}, \Lambda_{j}, \Lambda_{k}, \Lambda_{l}, \Lambda_{m}$, these sphere conditions are also independent of the leg lengths, as the Euler parameters fulfill $N=0$ (cf. Eq. (2)). Therefore the remaining Study parameters, which are computed from these equations, are also independent of the leg lengths. Therefore $\mathscr{B}_{n}$ depends on the geometry of the 5-legged manipulator and not on $R_{i}, R_{j}, R_{k}, R_{l}, R_{m}$. Based on this result the proof for the general case is closed by Definition 2 .

A similar argumentation also holds for the two special cases given in Remark 4.

Due to this theorem, we can choose arbitrary values for $R_{i}(i=1, \ldots, 6)$ for the computation of the bonds. This property can be used to simplify the sphere constraints $\Lambda_{i}$ of Eq. (2) by setting $R_{i}^{2}=a_{i}^{2}+b_{i}^{2}+c_{i}^{2}+A_{i}^{2}+B_{i}^{2}+C_{i}^{2}$.
We close this section with the following definition:

Definition 3. A bond is called singular, if it belongs to the vertex $E$ of the exceptional cone $N=0$.

\section{SG manipulators with self-motions}

In this section we discuss the use of bonds for the theory of self-motions of SG manipulators.

We assume that a given SG manipulator has a $d$ dimensional self-motion, which is not pure translational. As a $d$-dimensional self-motion corresponds with a $d$ dimensional solution of the direct kinematics problem, the seven quadrics $\Psi, \Lambda_{1}, \ldots, \Lambda_{6}$ have to have a $d$-dimensional set of points in common (= algebraic motion). But not all bonds of the bond-set $\mathscr{B}$ have to be implied by the selfmotion. This necessitates the following definition:

Definition 4. A bond, which does not belong to a $d$ dimensional $(d>0)$ algebraic motion $\not \subset N=0$ of the manipulator, is called pseudo-bond.

In order to improve the understanding of bonds and pseudo-bonds we give the following two examples with respect to the analogy of Remark 1 :

- Assume that $k_{1}, \ldots, k_{n}$ are conic sections located in pairwise distinct carrier planes, which have a line I in common. Moreover $k_{1}, \ldots, k_{n}$ are reducible, where each $k_{i}$ splits into the lines $\mathrm{I}$ and $\mathrm{I}_{i}$ with $\mathrm{I} \neq \mathrm{I}_{i}$ for $i=1, \ldots, n$. The common component I represents the self-motion and its ideal point is the corresponding bond.

- Assume that $k_{1}, \ldots, k_{n}$ are circles of arbitrary radius located in pairwise distinct carrier planes, which are parallel to each other. Nevertheless these circles do not have a component in common, they have the same two ideal points. Therefore these cyclic points correspond to pseudo-bonds.

Remark 5. In this context it should be noted that one can get rid of pseudo-bonds by using the following alternative definition of bonds for SG platforms (without the detour through 5-legged manipulators; cf. Definition 2):

$$
\begin{aligned}
\mathscr{B}:= & \operatorname{ZarClo}\left(V^{\star}\right) \cap\left\{\left(e_{0}: \ldots: f_{3}\right) \in P^{7} \mid\right. \\
& \left.\Psi, \Lambda_{1}, \Lambda_{2}, \Lambda_{3}, \Lambda_{4}, \Lambda_{5}, \Lambda_{6}, N=0\right\},
\end{aligned}
$$

where $V^{\star}$ denotes the variety $V$ after the removal of all components, which correspond to pure translational motions. $\diamond$

The restriction of the bond theory to non-translational self-motions does not cause any problems as all SG platforms, which possess pure translational self-motions, can easily be characterized as follows:

Theorem 2. A SG platform possesses a pure translational self-motion, if and only if the platform can be rotated about the center $\mathrm{m}_{1}=\mathrm{M}_{1}$ into a pose, where the vectors $\overrightarrow{\mathrm{M}}_{i} \mathrm{~m}_{i}$ for $i=2, \ldots, 6$ fulfill the condition $r k\left(\overrightarrow{\mathrm{M}_{2} \mathrm{~m}_{2}}, \ldots, \overrightarrow{\mathrm{M}_{6} \mathrm{~m}_{6}}\right) \leq 1$. 
Proof: Based on $\Lambda_{i}$ of Eq. (2) we prove this theorem analytically. Without loss of generality (w.l.o.g.) we can fix the orientation of the platform by $\left(e_{0}, e_{1}, e_{2}, e_{3}\right)=(1,0,0,0)$. As the group of translations is 3 -dimensional, we are looking for conditions, which imply at least a 1-dimensional variety of the ideal spanned by $\Psi, \Lambda_{1}, \Lambda_{2}, \Lambda_{3}$.

W.l.o.g. we can choose a fixed frame in a way that $M_{1}$ coincides with the origin, $\mathrm{M}_{2}$ is located on its $x$-axis, and $\mathrm{M}_{3}$ lies within the $x y$-plane $\left(\Rightarrow A_{1}=B_{1}=B_{2}=C_{1}=C_{2}=C_{3}=\right.$ $0)$. For the moving frame we can only assume w.l.o.g. that $\mathrm{m}_{1}$ coincides with its origin $\left(\Rightarrow a_{1}=b_{1}=c_{1}=0\right)$.

Then we can solve the system of equations $\Psi, \Delta_{2,1}, \Delta_{3,1}$, which is linear in $f_{0}, \ldots, f_{3}$, for:

1. $f_{0}, f_{2}, f_{3}$ if $D_{1} \neq 0$ holds with $D_{1}:=c_{2} b_{3}-c_{2} B_{3}-c_{3} b_{2}$,

2. $f_{0}, f_{1}, f_{3}$ if $D_{2} \neq 0$ holds with $D_{2}:=c_{2} a_{3}-c_{2} A_{3}-$ $c_{3} a_{2}+c_{3} A_{2}$,

3. $f_{0}, f_{1}, f_{2}$ if $D_{3} \neq 0$ holds with $D_{3}:=b_{2} A_{3}-b_{2} a_{3}-$ $b_{3} A_{2}+b_{3} a_{2}-B_{3} a_{2}+B_{3} A_{2}$,

and plug them into $\Lambda_{1}$. We denote the resulting expressions by $\Theta_{1}, \Theta_{2}$ and $\Theta_{3}$, respectively. In order to get a self-motion, $\Theta_{i}$ has to be fulfilled independently of $f_{i}$. Therefore the coefficient $\Xi_{i}$ of $f_{i}^{2}$ of $\Theta_{i}$, which only depends on the geometry of the manipulator and not on $R_{1}, R_{2}, R_{3}$, has to vanish.

We can solve $\Xi_{1}$, which is quadratic in $a_{2}$, for this variable if $T_{1} \neq 0$ holds with $T_{1}:=\left(b_{3}-B_{3}\right)^{2}+c_{3}^{2}$. Now it can easily be seen (e.g. by using the simplify command within Maple) that the obtained value for $a_{2}$ in dependency of $b_{2}, c_{2}, a_{3}, b_{3}, c_{3}, A_{2}, A_{3}, B_{3} \in \mathbb{R}$ cannot be a real number. This already yields the contradiction.

Analogously this can be done for $\Xi_{2}$ with respect to $b_{2}$, if $T_{2} \neq 0$ holds with $T_{2}:=\left(a_{3}-A_{3}\right)^{2}+c_{3}^{2}$, and for $\Xi_{3}$ with respect to $c_{2}$, if $T_{3} \neq 0$ holds with $T_{3}:=\left(a_{3}-A_{3}\right)^{2}+\left(b_{3}-\right.$ $\left.B_{3}\right)^{2}$.

Therefore we always end up with a contradiction as long as $D_{1} \neq 0 \neq T_{1}$ or $D_{2} \neq 0 \neq T_{2}$ or $D_{3} \neq 0 \neq T_{3}$ holds. Now it can easily be checked that $D_{1}=D_{2}=D_{3}=0$ equals the condition that the vectors $\overrightarrow{\mathrm{M}_{2} \mathrm{~m}_{2}}$ and $\overrightarrow{\mathrm{M}_{3} \mathrm{~m}_{3}}$ are linearly dependent ${ }^{4}$; i.e. $r k\left(\overrightarrow{\mathrm{M}_{2} \mathrm{~m}_{2}}, \overrightarrow{\mathrm{M}_{3} \mathrm{~m}_{3}}\right)=1$. In this case, the coefficient matrix of the linear system $\Psi, \Delta_{2,1}, \Delta_{3,1}$ has rank 2 . Then one of the radii $R_{1}, R_{2}, R_{3}$ can be determined in a way that the augmented coefficient matrix also has rank 2 . This already yields the 1-parametric translational self-motion.

Now it is not difficult to verify that all other combinatorial cases $X_{1}=Y_{2}=Z_{3}=0$ for $X, Y, Z \in\{D, T\}$ and $(X, Y, Z) \neq(D, D, D)$ also imply $D_{1}=D_{2}=D_{3}=0$. Therefore these cases are only special cases of the already discussed one, which also include the case that $\overrightarrow{\mathrm{M}_{2} \mathrm{~m}_{2}}$ and $\overrightarrow{\mathrm{M}_{3} \mathrm{~m}_{3}}$ equal the zero-vector $\mathbf{o}$.

Similar considerations for the ideals $\Psi, \Lambda_{1}, \Lambda_{i}, \Lambda_{j}$ for pairwise distinct $i, j \in\{2, \ldots, 6\}$ imply the condition $r k\left(\overrightarrow{\mathrm{M}_{2} \mathrm{~m}_{2}}, \ldots, \overrightarrow{\mathrm{M}_{6} \mathrm{~m}_{6}}\right) \leq 1$.

Finally it should be noted that all 1-parametric selfmotions are circular translations, which can easily be seen by considering a normal projection of the SG manipulator

${ }^{4}$ We denote the ideal point of this direction by $\mathrm{P}$ in direction of $\mathrm{P}$ (cf. footnote 4). Clearly we only get a 2parametric translational self-motion, if $P$ is not defined as $\overrightarrow{\mathrm{M}_{2} \mathrm{~m}_{2}}=\ldots=\overrightarrow{\mathrm{M}_{6} \mathrm{~m}_{6}}=\mathbf{o}$ holds $(\Rightarrow$ the platform and the base are congruent). This completes the discussion of SG platforms with pure translational self-motions.

Remark 6. This also verifies that translational self-motions correspond with components of $V_{n}$ (cf. Definition 1).

\subsection{Basic facts, results and examples}

We want to start this section with a first example; namely the Schönflies Borel Bricard motion.

Example 1. For the study of this self-motion we can assume w.l.o.g. that the platform and the base are both planar (cf. [15]); i.e. $c_{i}=C_{i}=0$ for $i=1, \ldots, 6$. If the base anchor points and the corresponding platform anchor points are coupled by an inversion with respect to the unit circle; i.e.

$$
A_{i}=a_{i} /\left(a_{i}^{2}+b_{i}^{2}\right), \quad B_{i}=b_{i} /\left(a_{i}^{2}+b_{i}^{2}\right),
$$

then there exists a 1-parametric self-motion with

$$
R_{i}^{2}:=\left(a_{i}^{2}+b_{i}^{2}+1\right)^{2} /\left(a_{i}^{2}+b_{i}^{2}\right)
$$

It can easily be verified that this self-motion is given by:

$$
\left(e_{0}: 0: 0: \pm e_{0} \sqrt{e_{0}^{2}-f_{3}^{2}} / f_{3}: \mp \sqrt{e_{0}^{2}-f_{3}^{2}}: 0: 0: f_{3}\right) .
$$

As it is well known [4] that all points of the platform move on spherical path, we can choose any six platform anchor points. We use the following ones:

$$
\begin{array}{lll}
\mathbf{m}_{1}=(17,-11)^{T}, & \mathbf{m}_{2}=(0,1)^{T}, & \mathbf{m}_{3}=(2,1)^{T}, \\
\mathbf{m}_{4}=(3,5)^{T}, & \mathbf{m}_{5}=(-7,13)^{T}, & \mathbf{m}_{6}=(1,0)^{T} .
\end{array}
$$

Then we run the algorithm given in Section 2. We compute $G C D_{0}$ and $G C D_{1}$ and their greatest common divisor, which equals $e_{0}^{2}+e_{3}^{2}$. Therefore we have to distinguish two cases:

1. $e_{0}=e_{3} I$, where I denotes the complex unit: Now the stepwise back-substitution can be done as follows. We set $e_{0}=e_{3} I$ in the already computed $L_{x}$ and $U_{i}$. Then we compute their common solution, which yields $e_{2}=0$. We proceed by plugging $e_{0}=e_{3} I$ and $e_{2}=0$ into the already computed $G_{x}$ and $F_{i}$ as well as $N$. The common solution of these equations is trivially given by $e_{1}=0$. Now we can compute $f_{0}, f_{1}, f_{2}$ from e.g. $\Psi, \Delta_{2,6}, \Delta_{3,6}$ which yields $f_{0}=f_{3} I$ and $f_{1}=f_{2}=0$. As $\Delta_{1,6}, \Delta_{4,6}, \Delta_{5,6}$ are fulfilled identically, we only remain with $\Lambda_{6}=4 e_{3}^{2}$, which implies $e_{3}=0$. This yields the first bond.

2. $e_{0}=-e_{3} I$ : Analogous computations yield the conjugated bond. 
As the set of bonds only consists of one element up to conjugation of coordinates; i.e.

$$
\mathscr{B}:=\{(0: 0: 0: 0: I: 0: 0: 1)\}
$$

the set $\mathscr{B}$ does not contain a pseudo-bond. Moreover as the bond has the expected dimension $d-1$, it corresponds to a point on the Study quadric $\Psi$. Note that this point is located in the linear subspace $E$ of $\Psi$, and therefore the bond is a singular one (cf. Definition 3).

Clearly the complex algebraic variety $\mathscr{B}$ can be used to classify all already known SG platforms with nontranslational self-motions with respect to algebraic properties of their bond-set. Note that these algebraic properties (e.g. algebraic multiplicities of bonds) have to be invariant with respect to changes of the reference frames in the platform and the base, respectively. In this context the well-known fact should be noted that the quadric $N=0$ and the linear subspace $E$ possess this invariance property (otherwise the presented bond theory and the notion of singular bonds would be senseless). This invariance property also holds for the following theorem, which follows immediately by intersecting the sphere constraint $\Lambda_{i}$ of Eq. (2) with $E$ :

Theorem 3. A singular bond fulfills the condition $f_{0}^{2}+$ $f_{1}^{2}+f_{2}^{2}+f_{3}^{2}=0$.

Remark 7. It is clear that non-singular bonds cannot be real as they fulfill $N=0$. Due to Theorem 3 this property also holds for singular bonds.

In the following we investigate a trivial self-motion, which is very interesting from the viewpoint of bond theory.

Example 2. We assume that $\mathrm{M}_{1}, \ldots, \mathrm{M}_{4}$ are located on a line g. Then there exists a trivial self-motion, if $\mathrm{m}_{5}$ and $\mathrm{m}_{6}$ are located on $\mathrm{g}$ as well, as in this case the platform can rotate around $\mathrm{g}$. This pure rotational self-motion is also known as butterfly self-motion (cf. [12]). W.l.o.g. we can assume that $\mathrm{g}$ is the $x$-axis of the fixed frame $\left(\Rightarrow B_{1}=\ldots=\right.$ $B_{4}=C_{1}=\ldots=C_{4}=0$ ) and that $\mathrm{m}_{5}$ and $\mathrm{m}_{6}$ are located on the $x$-axis of the moving frame $\left(\Rightarrow b_{5}=b_{6}=c_{5}=c_{6}=0\right)$. Moreover we can assume that the origin of the moving frame equals the point $(2 p, 0,0)^{T}$ of the fixed axis. We are still left with two possibilities, namely if the $x$-axes of the fixed and moving frame have the same orientation or the opposite one. Now this choice fixes the values of the radii $R_{1}(p), \ldots, R_{6}(p)$ in dependency of $p$. Then the Study parametrization of the rotation for equally oriented $x$-axes can for example be written as:

$$
\left(e_{0}: e_{1}: 0: 0:-e_{1} p: e_{0} p: 0: 0\right)
$$

By computing the bonds analogously to Example 1 we end up with the following set of bonds up to conjugation of coordinates:

$$
\begin{aligned}
\mathscr{B}:=\{ & (u I: u: 0: 0: v I: v: 0: 0), \\
& (0: 0: u: u I: 0: 0: v: v I)\} .
\end{aligned}
$$
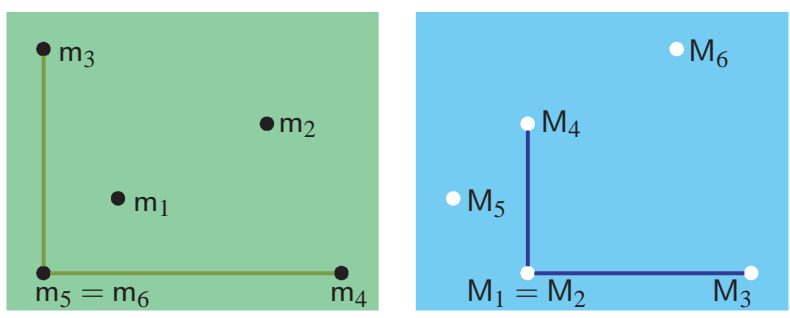

Fig. 2. Sketch of the platform (left) and the base (right) of the planar SG manipulator of Example 3, where $M_{1}=M_{2}$ and $m_{5}=m_{6}$ hold.

We get two 1-parametric bonds, where the ratio $u: v$ can be seen as projective parameter with $(u, v) \neq(0,0)$. For $u=0$ we get the singular points of these two 1-parametric bonds.

For each $p$ there exist two rotational self-motions (equally or oppositely oriented $x$-axes), which shows that the bonds have to be 1-dimensional. Therefore the first bond corresponds to the 1-dimensional set of rotational self-motions, where the $x$-axes have the same orientation. For the second bond they have opposite orientation.

This example shows very well the property formulated in Theorem 1, namely that bonds are independent of the leg lengths $R_{1}, \ldots, R_{6}$. Due to this property, bonds can not only be used for the above mentioned classification of known SG manipulators with self-motions, but they are also suited for the following two interesting tasks:

Task 1. Check if a given SG platform is free of nontranslational self-motions.

Task 2. Determine the geometry of SG platforms with nontranslational self-motions.

Before we discuss these tasks in the next two sections, we want to study a further very informative example from the viewpoint of bond theory, which is strongly connected with the previous example.

Example 3. The geometry of the planar SG manipulator, which is displayed in Fig. 2, is given by:

$$
\begin{array}{lll}
\mathbf{M}_{1}=(0,0)^{T}, & \mathbf{M}_{2}=(0,0)^{T}, & \mathbf{M}_{3}=(3,0)^{T}, \\
\mathbf{M}_{4}=(0,2)^{T}, & \mathbf{M}_{5}=(-1,1)^{T}, & \mathbf{M}_{6}=(2,3)^{T}, \\
\mathbf{m}_{1}=(1,1)^{T}, & \mathbf{m}_{2}=(3,2)^{T} & \mathbf{m}_{3}=(0,3)^{T}, \\
\mathbf{m}_{4}=(4,0)^{T}, & \mathbf{m}_{5}=(0,0)^{T}, & \mathbf{m}_{6}=(0,0)^{T} .
\end{array}
$$

An analogous computation as done for Example 1 yields the following set of bonds up to conjugation of coordinates:

$$
\begin{aligned}
\mathscr{B}:=\{ & (0: 0: u: u I: 0: 0: v: v I), \\
& (u I: u: 0: 0: v I: v: 0: 0), \\
& (0: u I: 0: u: 0: v I: 0: v), \\
& (u: 0: u I: 0: v: 0: v I: 0)\} .
\end{aligned}
$$

Due to Example 2, we can already give a geometric interpretation of these bonds. The first and second bond contain the 

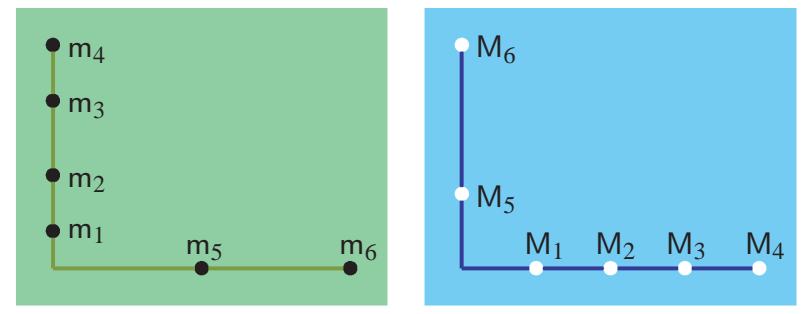

Fig. 3. Sketch of the platform (left) and the base (right) of a planar SG manipulator with pairwise distinct anchor points, where $B_{i}=$ $a_{i}=0$ for $i=1, \ldots, 4$ and $A_{5}=A_{6}=b_{5}=b_{6}=0$ hold.

information that the manipulator possesses rotational selfmotions around the $x$-axis of the fixed frame. These motions have the property that the points $\mathrm{m}_{5}=\mathrm{m}_{6}$ and $\mathrm{m}_{4}$ are located on the $x$-axis of the fixed frame.

An analogous interpretation can be given for the bonds three and four, as they also correspond to rotational selfmotions around the $y$-axis of the fixed frame. In this case, the points $\mathrm{m}_{5}=\mathrm{m}_{6}$ and $\mathrm{m}_{3}$ are located on the $y$-axis of the fixed frame.

Beside these butterfly self-motions, the manipulator is known to have also a spherical self-motion if $\mathrm{M}_{1}=\mathrm{M}_{2}$ coincides with $\mathrm{m}_{5}=\mathrm{m}_{6}$. Therefore this spherical self-motion is also encoded within $\mathscr{B}$ (cf. the later given Remark 9), which demonstrates that the set of bonds is more than the sum of its single bonds.

This example also shows that for a serious classification of SG manipulators with non-translational self-motions the consideration of algebraic multiplicities of bonds is essential, as there exist manipulators with the same set $\mathscr{B}$ of bonds given in Eq. (5), which only possess the butterfly motions but no spherical self-motion. As example we can consider the planar manipulator given in Fig. 3.

For the sake of length and simplicity we ignore the concept of algebraic multiplicities within this introduction article of bonds and postpone it to a follow-up paper.

\section{Study of task 1}

For the explanation given in the following two paragraphs we restrict ourself to 1-dimensional self-motions, but it should be noted that analogous considerations can be done for higher-dimensional ones as well.

The classical approach for the explicit solution of this problem is as follows: By eliminating the Study parameters according to the algorithm of Husty [13] we end up with a homogeneous polynomial $H$ of degree 40 in two Euler parameters, which additionally depends on $R_{1}, \ldots, R_{6}$. A necessary condition for a 1-parametric self-motion is that $H$ vanishes independently of these two Euler parameters. This implies a set of conditions for the unknowns $R_{1}, \ldots, R_{6}$, which can only be solved, if there exists a 1-parametric self-motion.

The problem with this method is that $H$ cannot be computed in dependency of $R_{1}, \ldots, R_{6}$ in the general case due to its complexity. Moreover, if this is possible for manipulators with special geometries, the solution of the set of equations is still not trivial at all.

With the bond theory task 1 simplifies considerably, as the following theorem holds due to the definition of bonds:

Theorem 4. A SG platform is free of non-translational selfmotions if $\mathscr{B}=\varnothing$ holds.

As due to Theorem 1 the computation of $\mathscr{B}$ is independent of all leg lengths, we get the paradoxical situation that task 1 can be solved independently of $R_{1}, \ldots, R_{6}$. In this context, we study the following examples:

Example 4. We consider a SG platform with generic geometry; i.e. the coordinate vectors $\mathbf{M}_{i}$ and $\mathbf{m}_{i}(i=1, \ldots, 6)$ of the anchor points $\mathrm{M}_{i}$ and $\mathrm{m}_{i}$, respectively, are generated randomly. By a straightforward computation according to the procedure of Section 2 it can easily be checked that $\bigcap_{i=1}^{6} \mathscr{B}_{i}=\varnothing$ holds. Moreover due to Theorem 2 a generic $S G$ platform is also free of pure translational self-motions. Therefore a generic SG manipulator does not possess any self-motions.

In the following we discuss one of the two special cases mentioned in Remark 4.

Example 5. The planar platform and planar base of the $S G$ manipulator are related by an affinity. Moreover we assume that the affinity is regular, as otherwise we end up with a trivial case of an architecturally singular manipulator, which is not of interest in this context. It was proven in [16] by geometric considerations that these so-called planar affine $S G$ manipulators only possess translatory self-motions, if they are not architecturally singular; i.e. the anchor points are not located on a conic section. In the following we want to verify this result by means of bond theory.

Due to a well known result (cf. [16-18]), we can use any six platform and base anchor points related by the affinity as long as they are not located on a conic section. We choose the following six platform anchor points:

$$
\begin{array}{lll}
\mathbf{m}_{1}=(0,0)^{T}, & \mathbf{m}_{2}=(1,0)^{T}, & \mathbf{m}_{3}=(1,1)^{T} \\
\mathbf{m}_{4}=(2,1)^{T}, & \mathbf{m}_{5}=(0,2)^{T}, & \mathbf{m}_{6}=(3,-2)^{T} .
\end{array}
$$

W.l.o.g. we can assume that $\mathrm{M}_{1}$ is located in the origin of the fixed frame and $\mathrm{M}_{2}$ on its $x$-axis. This already implies that the affinity is of the form $\mathbf{M}_{i}=\mathbf{A m}_{i}$ for $i=1, \ldots, 6$, where the $2 \times 2$ transformation matrix $\mathbf{A}$ is given by:

$$
\mathbf{A}:=\left(\begin{array}{cc}
\alpha_{1} & \alpha_{2} \\
0 & \alpha_{3}
\end{array}\right)
$$

with $\operatorname{det}(\mathbf{A})=\alpha_{1} \alpha_{3} \neq 0$. As for this $S G$ manipulator any four equations of the set $\mathscr{I}_{0}$ of Eq. (3) are linearly dependent, the algorithm of Section 2 fails. In this case we use the following modified one:

Now we can solve $\Psi, \Delta_{j, i}, \Delta_{k, i}$ for three of the four $f_{i}$ 's. The remaining $f_{i}$ can be computed from $\Lambda_{i}$, but this is not of 
interest. If we plug the obtained expression for the three $f_{i}$ 's into $\Delta_{l, i}, \Delta_{m, i}$, we get homogeneous quadratic equations in the Euler parameters. Therefore they represent two quadrics in the Euler parameter space $P^{3}$. Moreover we denote the intersection curve of these quadrics by $K_{l, m}$. Now the intersection of $K_{l, m}$ with $N=0$ can be computed as follows:

We eliminate an Euler parameter $e_{s}$ by computing the resultant $\operatorname{Res}\left(\Delta_{l, i}, N, e_{s}\right)$ and $\operatorname{Res}\left(\Delta_{m, i}, N, e_{s}\right)$. Then we eliminate a further Euler parameter $e_{t}$ by computing the resultant $J_{l, m}$ of the obtained two expressions with respect to $e_{t}$.

If we start with another system of linear equations and proceed analogously, then we end up with another curve. In this way we can generate the following six curves:

$$
K_{j, k}, \quad K_{j, l}, \quad K_{j, m}, \quad K_{k, l}, \quad K_{k, m}, \quad K_{l, m}
$$

Therefore the bonds of the 5-legged manipulator correspond with the greatest common divisor $G C D_{n}$ of

$$
J_{j, k}, \quad J_{j, l}, \quad J_{j, m}, \quad J_{k, l}, \quad J_{k, m}, \quad J_{l, m} .
$$

Finally the bonds of the planar affine SG manipulator are implied by the greatest common divisor of $G C D_{1}, \ldots, G C D_{6}$. In our case this yields $\alpha_{1} \alpha_{3}$, which cannot vanish without contradiction. Therefore $\mathscr{B}=\varnothing$ holds, which proves again that planar affine SG manipulators can only have translational self-motions, if they are not architecturally singular.

For the sake of completeness it should be noted that these manipulators only have translational self-motions, if and only if the singular values $s_{1}$ and $s_{2}$ of $\mathbf{A}$ with $0<s_{1} \leq s_{2}$ fulfill the relation $s_{1} \leq 1 \leq s_{2}$. For more details see [16]. $\diamond$

Remark 8. For the second special case of Remark 4, where the platform and the base are congruent, the set $\mathscr{B}$ of bonds can be computed as in Example 5.

Note that due to the possibility of pseudo-bonds and bonds of complex self-motions we cannot replace "if" by "if and only if" in Theorem 4. In order to point this out more clearly, we study the self-motional behavior of a superset of planar affine SG platforms.

Example 6. We investigate so-called planar projective $S G$ platforms; i.e. corresponding anchor points of the planar platform and planar base are coupled by a non-singular projectivity $\kappa: \mathrm{m}_{i} \mapsto \mathrm{M}_{i}$ for $i=1, \ldots, 6$. Moreover due to Example 5 we can assume that $\kappa$ is no affinity. The author has proven within the publications $[16,19,20]$ that these manipulators are free of self-motions (over $\mathbb{R}$ ), if they are not architecturally singular; i.e. if the anchor points are not located on a conic section.

According to [19] the direct kinematics of these manip- ulators is determined by the following set of equations:

$$
\begin{aligned}
& \Delta_{2,1}:=t_{2}+R_{2} N=0, \\
& \Delta_{3,1}:=\bar{t}_{1}+R_{3} N=0, \\
& \Delta_{4,1}:=r_{12}-R_{4} N=0, \\
& \Delta_{5,1}:=t_{1}+t_{2}+R_{5} N+\beta\left(r_{12}+r_{22}\right)=0, \\
& \Delta_{6,1}:=\bar{t}_{1}+\bar{t}_{2}+R_{6} N-\left(r_{11}+r_{12}\right)=0,
\end{aligned}
$$

with

$$
\begin{aligned}
& \bar{t}_{1}:=2\left(e_{0} f_{1}-e_{1} f_{0}-e_{2} f_{3}+e_{3} f_{2}\right), \\
& \bar{t}_{2}:=2\left(e_{0} f_{2}+e_{1} f_{3}-e_{2} f_{0}-e_{3} f_{1}\right) .
\end{aligned}
$$

Moreover $\beta \in \mathbb{R} \backslash\{0\}$ is the parameter of the projectivity $\kappa$. The remaining missing condition $\Lambda_{1}$ can be computed according to Eq. (2) for $\mathbf{m}_{1}=(-\beta, 0,0)^{T}$ and $\mathbf{M}_{1}=$ $(0,-1,0)^{T}$, respectively.

For the given set of equations we run the procedure of Section 2, which finally yields the following set ${ }^{5}$ of bonds up to conjugation of coordinates:

$$
\begin{aligned}
\mathscr{B}:=\{ & (0: 0: 0: 0: I: 1: 1:-I), \\
& (0: 0: 0: 0: I: 1:-1: I), \\
& (0: 0: 0: 0: \mu I: \mu: 1: I), \\
& (0: 0: 0: 0: \mu I: 1: \mu:-I), \\
& (0: 0: 0: 0: v I: 1:-v: I), \\
& (0: 0: 0: 0: v I: v:-1:-I)\},
\end{aligned}
$$

with $\mu:=\sqrt{2}+1$ and $v:=\sqrt{2}-1$. Due to the above cited result, the six singular bonds are either pseudo-bonds or they belong to complex self-motions of the manipulator.

Until now it is an open problem how to identify bonds of real self-motions only from properties of the bond-set. The answering of this question is dedicated to future research.

\section{Study of task 2}

In this section we show that the bond theory can also be used to design parallel manipulators with self-motions. In this context we provide a simple proof for the following theorem on the spherical analogs of SG manipulators (i.e. spherical three degrees of freedom (dof) mechanisms, where the platform is connected via three RPR-legs with the base), which was presented in [16]:

Theorem 5. Given is a spherical 3-dof RPR manipulator, where the base anchor points $\mathrm{M}_{1}^{\circ}, \mathrm{M}_{2}^{\circ}, \mathrm{M}_{3}^{\circ}$ and platform anchor points $\mathrm{m}_{1}^{\circ}, \mathrm{m}_{2}^{\circ}, \mathrm{m}_{3}^{\circ}$ are located on great circles (cf. Fig. 4, left). This manipulator has a self-motion, if and only if two platform anchor points or two base anchor points coincide (assumed that antipodal points are identified). 

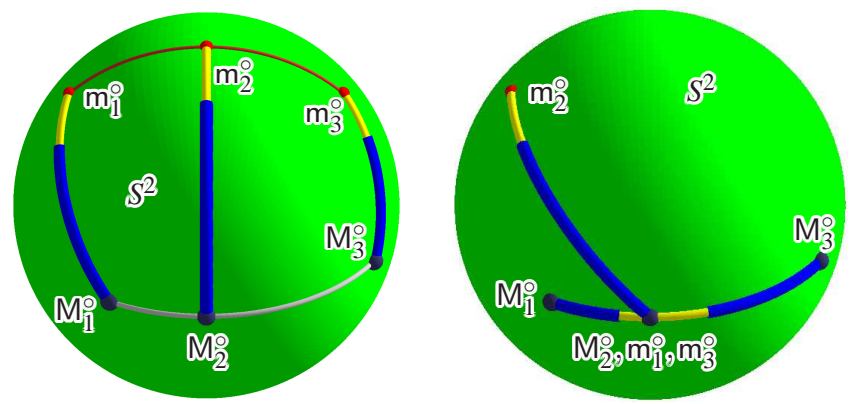

Fig. 4. Left: Spherical 3-dof RPR manipulator. Right: W.I.o.g. we can assume that $\mathrm{m}_{1}^{\circ}$ and $\mathrm{m}_{3}^{\circ}$ coincide (after a perhaps necessary exchange of the platform and the base and reindexing of anchor points). In the configuration, where $\mathrm{m}_{1}^{\circ}=\mathrm{m}_{3}^{\circ}$ coincides with $\mathrm{M}_{2}^{\circ}$, the platform has a trivial rotational self-motion.

Proof: W.1.o.g. we can assume that the radius of the sphere $S^{2}$ equals one and that this unit-sphere is centered in the origin of the fixed frame. Moreover we can assume w.1.o.g. that $\mathrm{M}_{1}^{\circ}$ is located on the $x$-axis of the fixed frame and that $M_{2}^{\circ}, M_{3}^{\circ}$ belongs to the $x y$-plane of the fixed frame.

W.l.o.g. we can assume for the platform that $m_{1}^{\circ}$ is located on the $y$-axis of the moving frame and that $\mathrm{m}_{2}^{\circ}, \mathrm{m}_{3}^{\circ}$ belongs to the $x y$-plane of the moving frame. By using the half-angle substitution we can set:

$$
\begin{array}{ll}
\mathbf{M}_{1}^{\circ}=(1,0,0)^{T}, & \mathbf{M}_{i}^{\circ}=\left(\frac{2 Q_{i}}{1+Q_{i}^{2}}, \frac{1-Q_{i}^{2}}{1+Q_{i}^{2}}, 0\right)^{T}, \\
\mathbf{m}_{1}^{\circ}=(0,1,0)^{T}, & \mathbf{m}_{i}^{\circ}=\left(\frac{1-q_{i}^{2}}{1+q_{i}^{2}}, \frac{2 q_{i}}{1+q_{i}^{2}}, 0\right)^{T},
\end{array}
$$

with $q_{i}, Q_{i} \in \mathbb{R}$ for $i=2,3$. Now we can compute the three leg constraints $\Lambda_{1}, \Lambda_{2}$ and $\Lambda_{3}$ according to Eq. (2) under consideration of $f_{0}=f_{1}=f_{2}=f_{3}=0$ (cf. [21]).

In general this 3-legged manipulator has no selfmotions. Therefore we consider the motion of the manipulator after the removal of the $j$ th leg. Note that this yields a spherical 4-bar mechanism, which trivially has only nontranslational self-motions (= spherical coupler motions). Now the set $\mathscr{B}_{j}$ of bonds of this spherical 4-bar mechanism consists of the common points of $\Lambda_{k}, \Lambda_{l}$ and $N=0$ with pairwise distinct $j, k, l \in\{1,2,3\}$. These points can be computed as follows:

We eliminate an Euler parameter $e_{s}$ by computing $S_{1}:=$ $\operatorname{Res}\left(\Lambda_{k}, N, e_{s}\right), S_{2}:=\operatorname{Res}\left(\Lambda_{l}, N, e_{s}\right)$ and $S_{3}:=\operatorname{Res}\left(\Lambda_{k}, \Lambda_{l}, e_{s}\right)$. Then we can eliminate a further Euler parameter $e_{t}$ by calculating $\operatorname{Res}\left(S_{1}, S_{2}, e_{t}\right), \operatorname{Res}\left(S_{1}, S_{3}, e_{t}\right)$ and $\operatorname{Res}\left(S_{2}, S_{3}, e_{t}\right)$. The greatest common divisor $G C D_{j}$ of the obtained three expressions corresponds with the solution of our problem. The set of bonds can again be computed by a stepwise backsubstitution. E.g. we get the following set $\mathscr{B}_{3}$ of bonds up

\footnotetext{
${ }^{5}$ It is worth noting that $\mathscr{B}$ does not depend on the parameter $\beta$, which determines the geometry of the planar projective SG platform (cf. $[19,20]$ ).
}

to conjugation of coordinates:

$$
\begin{aligned}
\mathscr{B}_{3}:=\{ & \left(q_{2} I:-q_{2}: 1: I: 0: 0: 0: 0\right), \\
& \left(I: 1: q_{2}:-q_{2} I: 0: 0: 0: 0\right), \\
& \left(Q_{2}: I:-Q_{2} I: 1: 0: 0: 0: 0\right), \\
& \left.\left(1: Q_{2} I: I:-Q_{2}: 0: 0: 0: 0\right)\right\} .
\end{aligned}
$$

A necessary condition for a self-motion of the spherical 3dof RPR manipulator is that the sets $\mathscr{B}_{1}, \mathscr{B}_{2}$ and $\mathscr{B}_{3}$ have a common bond, which is equivalent with the statement that $G C D_{1}, G C D_{2}$ and $G C D_{3}$ have a common factor.

It is well known that $G C D_{i}$ and $G C D_{j}$ only have a common factor, if the resultant $P_{i, j}$ of these two expressions with respect to one of the two remaining Euler parameters vanishes. Therefore we have to compute the greatest common divisor of $P_{1,2}, P_{1,3}$ and $P_{2,3}$, which factors into:

$$
\begin{aligned}
& \left(q_{2}^{2}-1\right)\left(q_{3}^{2}-1\right)\left(Q_{2}^{2}-1\right)\left(Q_{3}^{2}-1\right) \\
& \left(q_{2}-q_{3}\right)\left(Q_{2}-Q_{3}\right)\left(1+q_{2} q_{3}\right)\left(1+Q_{2} Q_{3}\right)
\end{aligned}
$$

beside the remaining Euler parameter. But by setting this Euler parameter equal zero, the stepwise back-substitution yields $e_{0}=e_{1}=e_{2}=e_{3}=0$; a contradiction. Moreover it can easily be checked that the vanishing of any factor of Eq. (7) implies the coincidence of two platform anchor points or base anchor points, if we identify antipodal points. This condition is already sufficient, as such manipulators always have a pure rotational self-motion (cf. Fig. 4, right).

Remark 9. We consider the spherical coupler motion, which results from the removal of the third leg. If we set $q_{2}=Q_{2}=0$, it can easily be seen that this motion is equivalent with the spherical motion of the SG manipulator of Example 3. For $(u, v)=(1,0)$ the set $\mathscr{B}$ of Eq. (5) equals $\mathscr{B}_{3}$ of Eq. (6). This demonstrates that the spherical self-motion of the $S G$ manipulator is indeed encoded within the set $\mathscr{B} . \diamond$

It the following we show that by means of bond theory even a stronger statement can be proven in a very elegant way.

Theorem 6. Theorem 5 also holds if the anchor points are not assumed to be located on great circles.

Proof: First of all we have to generalize the coordinates of the third pair of anchor points as follows:

$$
\begin{aligned}
& \mathbf{M}_{3}^{\circ}=\left(\frac{2 Q_{3}\left(1-Q_{4}^{2}\right)}{\left(1+Q_{3}^{2}\right)\left(1+Q_{4}^{2}\right)}, \frac{\left(1-Q_{3}^{2}\right)\left(1-Q_{4}^{2}\right)}{\left(1+Q_{3}^{2}\right)\left(1+Q_{4}^{2}\right)}, \frac{2 Q_{4}}{1+Q_{4}^{2}}\right)^{T}, \\
& \mathbf{m}_{3}^{\circ}=\left(\frac{\left(1-q_{3}^{2}\right)\left(1-q_{4}^{2}\right)}{\left(1+q_{3}^{2}\right)\left(1+q_{4}^{2}\right)}, \frac{2 q_{3}\left(1-q_{4}^{2}\right)}{\left(1+q_{3}^{2}\right)\left(1+q_{4}^{2}\right)}, \frac{2 q_{4}}{1+q_{4}^{2}}\right)^{T} .
\end{aligned}
$$

In this case we do not proceed like in the proof of Theorem 5, as we want to present another way of arguing:

As the spherical 3-dof RPR manipulator can only have a self-motion, if its set of bonds is not empty, at least one bond 
of $\mathscr{B}_{3}$ of Eq. (6) has to fulfill the leg constraint $\Lambda_{3}$. If we plug the first bond of $\mathscr{B}_{3}$ into $\Lambda_{3}$, the numerator factors into:

$$
\begin{aligned}
& {\left[q_{4}\left(q_{3}-q_{2}\right)-\left(1+q_{2} q_{3}\right) I\right]\left[q_{4}\left(1+q_{2} q_{3}\right)+\left(q_{2}-q_{3}\right) I\right]} \\
& {\left[Q_{4}\left(Q_{3}-1\right)+\left(Q_{3}+1\right) I\right]\left[Q_{4}\left(Q_{3}+1\right)+\left(Q_{3}-1\right) I\right] .}
\end{aligned}
$$

The vanishing of the real and imaginary part of any factor already implies the coincidence of two anchor points, if we take $q_{i}, Q_{i} \in \mathbb{R}$ for $i=2,3,4$ into account. This can be checked analogously for the remaining bonds of $\mathscr{B}_{3}$.

Clearly the coincidence of two anchor points is again sufficient for the existence of a rotational self-motion (cf. Fig. 4, right), which proves the theorem.

Remark 10. It remains as an exercise for the reader to verify in an analogous way that planar 3-dof RPR manipulators only have non-translational self-motions, if two platform anchor points or two base anchor points coincide. Moreover it is a simple task to figure out that the platform and the base of planar 3-dof RPR manipulators with translatory self-motions have to be congruent.

If we want to design SG platforms with nontranslational self-motions, we can also make use of the necessary condition that these manipulators have to possess bonds. This is demonstrated on the basis of the following problem, which is connected with the one discussed by Duporcq in [22]:

We start with a 5-legged manipulator with planar platform and planar base. Every manipulator of this type has at least a 1-parametric self-motion. Now we are interested in a sixth point pair $\left(m_{6}, M_{6}\right)$ located in the planar platform and planar base in a way that the resulting planar SG platform has the same set of bonds, which correspond to self-motions as the given 5-legged manipulator. This is a necessary condition for the SG platform in order to possess the same self-motions as the 5-legged manipulator.

Due to the Definitions 1 and 2, this necessary condition is trivially fulfilled if $\Lambda_{6}$ is contained within the ideal spanned by $\Lambda_{1}, \ldots, \Lambda_{5}, \Psi$ as $V=V_{6}$ holds. Moreover this already implies that both manipulators have the same self-motions. As all involved equations are homogeneous quadratic in the Study parameters, there has to exist coefficients $\lambda_{1}, \ldots, \lambda_{6}$ with

$$
\sum_{i=1}^{5} \lambda_{i} \Lambda_{i}+\lambda_{6} \Psi-\Lambda_{6}=0
$$

As this equation has to be fulfilled independently of the Study parameters, all coefficients of these parameters have to vanish. As the monomials $e_{i} f_{i}$ for $i=0, \ldots, 3$ are only contained in $\Psi$, this already implies $\lambda_{6}=0$. Now we have an analogous problem to the one of [18], where the equation $\sum_{i=1}^{6} \lambda_{i} \Lambda_{i}-\Lambda_{7}=0$ was studied. Therefore we can proceed similar to the cited paper. By doing this we end up with the following set of necessary and sufficient equations (cf. Eq. (30) of [18]):

$$
\left(\mathbf{s}_{1}, \mathbf{s}_{2}, \mathbf{s}_{3}, \mathbf{s}_{4}, \mathbf{s}_{5}\right)\left(\lambda_{1}, \lambda_{2}, \lambda_{3}, \lambda_{4}, \lambda_{5}\right)^{T}=\mathbf{s}_{6}
$$

with $\mathbf{s}_{i}:=\left(1, a_{i}, b_{i}, A_{i}, B_{i}, a_{i} A_{i}, a_{i} B_{i}, b_{i} A_{i}, b_{i} B_{i}\right)^{T}$. This system only has a solution, if the rank of the $9 \times 5$ coefficient matrix $\mathbf{M}:=\left(\mathbf{s}_{1}, \mathbf{s}_{2}, \mathbf{s}_{3}, \mathbf{s}_{4}, \mathbf{s}_{5}\right)$ equals the rank of the augmented coefficient matrix $\mathbf{M}_{+}:=\left(\mathbf{s}_{1}, \mathbf{s}_{2}, \mathbf{s}_{3}, \mathbf{s}_{4}, \mathbf{s}_{5}, \mathbf{s}_{6}\right)$. We distinguish the following two cases:

(a) $r k(\mathbf{M})<5$ : Now the 5-legged manipulator is a so-called degenerated one (cf. [23]), which is only possible if a minimum of four anchor points is collinear [24]. Moreover it was shown by the author [24] that in this case at least a 1-dimensional solution of $r k(\mathbf{M})=r k\left(\mathbf{M}_{+}\right)$exists with respect to the unknowns $a_{6}, b_{6}, A_{6}, B_{6}$.

(b) $r k(\mathbf{M})=5$ : The solution of the problem $r k(\mathbf{M})=$ $r k\left(\mathbf{M}_{+}\right)=5$ is more complicated, as special cases have to be studied. But for the general case there exist an unique solution. For a complete and detailed discussion please see [24].

If a sixth point pair $\left(\mathrm{m}_{6}, \mathrm{M}_{6}\right)$ with $r k(\mathbf{M})=r k\left(\mathbf{M}_{+}\right)$exists, which therefore is a solution to Duprocq's problem, then the resulting SG platform is always architecturally singular, because $r k\left(\mathbf{M}_{+}\right)<6$ holds (cf. Remark 1 of [8]).

As a consequence we can also give the missing geometric/kinematic reasoning for Lemma 2 of [6], which was only proven analytically. This lemma reads as follows:

Lemma 1. Let a planar SG manipulator with no four anchor points collinear be architecturally singular. Then the collinearity of $\mathrm{m}_{i}, \mathrm{~m}_{j}, \mathrm{~m}_{k}$ implies the collinearity of $\mathrm{M}_{l}, \mathrm{M}_{m}, \mathrm{M}_{n}$ and vice versa with pairwise distinct $i, j, k, l, m, n \in\{1, \ldots, 6\}$.

Proof: As no four anchor points are collinear, the manipulator fulfills $r k\left(\mathbf{M}_{+}\right)=5$. Moreover the removal of any leg (w.l.o.g. we can assume that this is the leg with index 6) yields a 5-legged manipulator with $\operatorname{rk}(\mathbf{M})=5$. For this 5legged manipulator we can assume w.l.o.g. (after a possible necessary renumbering of indices and exchange of platform and base) that $\mathrm{M}_{1}, \mathrm{M}_{2}, \mathrm{M}_{3}$ are located on a line $\mathrm{g}$. Moreover we can choose coordinate systems in the base in a way that $g$ equals the $x$-axis of the fixed frame and that $\mathrm{m}_{4}$ and $\mathrm{m}_{5}$ are located on the $x$-axis of the moving frame.

Clearly the 5-legged manipulator has two 1-parametric sets of butterfly motions around the line g. This can also be verified by computing $\mathscr{B}_{6}$, which contains the two bonds of Eq. (4). Now the sphere constraint $\Lambda_{6}$ of the sixth leg has to contain these bonds as well. By plugging both bonds into Eq. (2) we get $\pm 4 b_{6} B_{6} u^{2}$. This confirms the trivial fact that we can only attach a sixth leg without disturbing these rotational self-motions, if $\mathrm{m}_{6}$ or $\mathrm{M}_{6}$ is located on the $x$-axis. The condition that no four anchor points are allowed to be collinear implies the collinearity of $\mathrm{m}_{4}, \mathrm{~m}_{5}, \mathrm{~m}_{6}$. 


\section{Conclusion and future work}

In this paper we transfered the basic idea of bonds for overconstrained closed chains with rotational joints, to the theory of self-motions of SG platforms. Clearly this concept is not limited to SG platforms, but it can also be adopted for other parallel manipulators as well [e.g. spherical 3-dof RPR manipulators (cf. Theorems 5 and 6) or planar 3-dof RPR manipulators (cf. Remark 10)].

We presented some basic facts and results on bonds and demonstrated the potential of this theory on the basis of several examples. Moreover we showed (cf. Example 3) that for a further deeper study of bonds, their algebraic multiplicities have to be considered as well. Based on this concept we want to read off more information from the bond-set; e.g. if a bond belongs to a real self-motion (cf. Example 6) and the degree of this self-motion.

Beside this, it is also planned to use the theory of bonds for the determination of necessary conditions for the design of non-architecturally singular SG platforms with selfmotions. This theory even raise the hope of giving a complete solution to the Borel Bricard problem in the future.

Due to the computation of bonds given in Section 2, it is obvious that they are also connected with the manipulators direct kinematic problem. Therefore this theory can also be of interest for the determination of the maximal number of solutions for the forward kinematics of manipulators with special geometries (e.g. coincidence of anchor points [25]).

\section{Achnowledgements}

This research is funded by Grant No. I 408-N13 of the Austrian Science Fund FWF within the project "Flexible polyhedra and frameworks in different spaces", an international cooperation between FWF and RFBR, the Russian Foundation for Basic Research. Moreover the author is supported by Grant No. P 24927-N25 for the FWF project "Stewart Gough platforms with self-motions".

Moreover the author would like to thank the reviewers for their useful comments and suggestions, which have helped to improve the quality of the paper.

\section{References}

[1] Hegedüs, G., Schicho, J., and Schröcker, H-P., 2012, "Bond Theory and Closed 5R Linkages", Latest Advances in Robot Kinematics (J. Lenarcic, M. Husty eds.), pp. 221-228.

[2] Hegedüs, G., Schicho, J., and Schröcker, H-P., 2013, "The theory of bonds: A new method for the analysis of linkages", Mechanism and Machine Theory, in press.

[3] Borel, E., 1908, "Mémoire sur les déplacements à trajectoires sphériques", Mémoire présenteés par divers savants à l'Académie des Sciences de l'Institut National de France 33(1) 1-128.

[4] Bricard, R., 1906, "Mémoire sur les déplacements à trajectoires sphériques", Journal de École Polytechnique 11 1-96.
[5] Husty, M., 2000, "E. Borel's and R. Bricard's Papers on Displacements with Spherical Paths and their Relevance to Self-Motions of Parallel Manipulators", Int. Symp. on History of Machines and Mechanisms (M. Ceccarelli ed.), Kluwer, pp. 163-172.

[6] Karger, A., 2003, "Architecture singular planar parallel manipulators", Mechanism and Machine Theory 38(11) 1149-1164.

[7] Nawratil, G., 2008, "On the degenerated cases of architecturally singular planar parallel manipulators", Journal for Geometry and Graphics 12(2) 141-149.

[8] Röschel, O., and Mick, S., 1998, "Characterisation of architecturally shaky platforms", Advances in Robot Kinematics: Analysis and Control (J. Lenarcic, M.L. Husty eds.), Kluwer, pp. 465-474.

[9] Wohlhart, K., 2010, "From higher degrees of shakiness to mobility", Mechanism and Machine Theory 45(3) 467-476.

[10] Karger, A., 2008, "Architecturally singular nonplanar parallel manipulators", Mechanism and Machine Theory 43(3) 335-346.

[11] Nawratil, G., 2009, "A new approach to the classification of architecturally singular parallel manipulators", Computational Kinematics (A. Kecskemethy, A. Müller eds.), Springer, pp. 349-358.

[12] Nawratil, G., 2012, "Review and recent results on Stewart Gough platforms with self-motions", Applied Mechanics and Materials 162 151-160.

[13] Husty, M.L., 1996, "An algorithm for solving the direct kinematics of general Stewart-Gough platforms", Mechanism and Machine Theory 31(4) 365-380.

[14] Husty, M., Karger, A., Sachs, H., and Steinhilper, W., 1997, "Kinematik und Robotik", Springer.

[15] Husty, M.L., and Karger, A., 2002, "Self motions of Stewart-Gough platforms: an overview", Proc. of the workshop on fundamental issues and future research directions for parallel mechanisms and manipulators (C.M. Gosselin, I. Ebert-Uphoff eds.), Quebec, Canada, pp. 131-141.

[16] Nawratil, G., 2012, "Self-motions of planar projective Stewart Gough platforms", Latest Advances in Robot Kinematics (J. Lenarcic, M. Husty eds.), Springer, pp. 27-34.

[17] Borras, J., Thomas, F., and Torras, C., 2010, "Singularity-invariant leg rearrangements in doublyplanar Stewart-Gough platforms", Proc. of Robotics Science and Systems, Zaragoza, Spain.

[18] Mielczarek, S., Husty, M.L., and Hiller, M., 2002, "Designing a redundant Stewart-Gough platform with a maximal forward kinematics solution set", In Proc. of the International Symposion of Multibody Simulation and Mechatronics, Mexico City, Mexico.

[19] Nawratil, G., 2013 "On elliptic self-motions of planar projective Stewart Gough platforms", Transactions of the Canadian Society for Mechanical Engineering 37 (1) in press.

[20] Nawratil, G., 2013, "Non-existence of planar projective Stewart Gough platforms with elliptic self- 
motions", Computational Kinematics (F. Thomas, A. Perez Garcia eds.), Springer, pp. 41-48.

[21] Brunnthaler, K., Schröcker, H-P., and Husty, M., 2006, "Synthesis of spherical four-bar mechanisms using spherical kinematic mapping", Advances in Robot Kinematics: Mechanisms and Motion (J. Lenarcic, B. Roth eds.), Springer, pp. 377-384.

[22] Duporcq, E., 1898, "Sur la correspondance quadratique et rationnelle de deux figures planes et sur un déplacement remarquable", Comptes Rendus des Séances de l'Académie des Sciences 126 1405-1406.

[23] Karger, A., 1998, "Architecture singular parallel manipulators", Advances in Robot Kinematics: Analysis and Control (J. Lenarcic, M.L. Husty eds.), Kluwer, pp. $445-454$.

[24] Nawratil, G., "Correcting Duporcq's theorem", under review.

[25] Faugère, J.C., and Lazard, D., 1995, "Combinatorial classes of parallel manipulators", Mechanism and Machine Theory 30(6) 765-776. 